\title{
Plasma Channel Diagnostic Based on Laser Centroid Oscillations
}

\author{
A. J. Gonsalves, K. Nakamura, C. Lin, J. Osterhoff, S. Shiraishi, C. B. Schroeder, C. G. \\ R. Geddes, Cs. Tóth, E. Esarey, and W. P. Leemans \\ Lawrence Berkeley National Laboratory, Berkeley, CA 94720, \\ $U S A$
}

(Dated: 12 February 2010)

A technique has been developed for measuring the properties of discharge-based plasma channels by monitoring the centroid location of a laser beam exiting the channel as a function of input alignment offset between the laser and the channel. Experiments were performed using low-intensity $\left(<10^{14} \mathrm{Wcm}^{-2}\right)$ laser pulses focused onto the entrance of a hydrogen-filled capillary discharge waveguide. Scanning the laser centroid position at the input of the channel and recording the exit position allows determination of the channel depth with an accuracy of a few $\%$, measurement of the transverse channel shape, and inference of the matched spot size. In addition, accurate alignment of the laser beam through the plasma channel is provided by minimizing laser centroid motion at the channel exit as the channel depth is scanned either by scanning the plasma density or the discharge timing. The improvement in alignment accuracy provided by this technique will be crucial for minimizing electron beam pointing errors in laser plasma accelerators. 


\section{INTRODUCTION}

Laser-driven plasma accelerators ${ }^{1}$ have shown acceleration gradients orders of magnitude higher than those found in conventional accelerators, offering the potential for a significant reduction in accelerator length and cost. Recent progress, such as the production of high-quality $\mathrm{GeV}$ electron beams in just a few $\mathrm{cm}^{2,3}$ has increased interest in laser-plasma accelerator technology as a driver for radiation sources - ranging from coherent $\mathrm{THz}^{4}$ to free electron laser (FEL) x-ray sources ${ }^{5}$ and Thomson scattering gamma ray sources ${ }^{6}$ - and as a path towards a TeV-class linear collider ${ }^{7}$.

For both radiation source and high energy physics applications, precise control over electron beam pointing is essential. Current state-of-the-art laser plasma accelerators (LPAs) have rms pointing fluctuation at or below the $1 \mathrm{mrad} \mathrm{level}^{8-10}$. Although this is tolerable for some applications, it is at least one order of magnitude larger than deemed necessary for light source or staging applications.

For a light source based on on-axis spontaneous radiation of an electron beam passing through an undulator, the radiation wavelength may change by more than several percent for pointing fluctuations of $1 \mathrm{mrad}$. In the case of a seeded FEL, overlap between the electron beam and radiation requires tight tolerances on electron beam pointing. Minimizing shotto-shot fluctuation in electron beam propagation angle is therefore an essential requirement for the successful operation of a light source.

In the case of an LPA-based high-energy collider, achieving high particle energy will require the use of multiple accelerator stages, whose alignment will be crucial to beam quality, and high efficiency. An example of a possible design has been described elsewhere ${ }^{7,11}$ in which each stage would consist of a meter-scale plasma channel, powered by high power

(> few $100 \mathrm{TW}$ ) laser pulses that are merged onto the path of the electron beam exiting from the previous stage. The distance between stages should be minimized to reduce the total length of the accelerator and for simplification of electron beam transport, and will be determined by the laser damage threshold of the optic used to couple the laser energy into the stage. Plasma mirror technology ${ }^{12}$ potentially reduces this distance to $\sim 10 \mathrm{~cm}$ offering the possibility for TeV energy gain ${ }^{7,11}$ with $\sim 100$ stages in just $\gtrsim 100 \mathrm{~m}$. Even at such distances however, 1 mrad pointing deviation would result in an offset of the electron beam at the subsequent stage by $100 \mu \mathrm{m}$. This is on the order of the transverse extent of the 
wakefield, and jitter significantly less than this will be required for a linear collider ${ }^{13}$.

Laser pointing errors are one potential source of electron beam pointing errors that must be controlled (others include laser mode asymmetry, pulse-front tilt ${ }^{14}$, and plasma gradients). Shot-to-shot fluctuations in laser pointing cause the laser centroid to be offset from the plasma channel and to undergo transverse oscillations about the channel axis. Electrons accelerated by the plasma wakefield will then undergo betatron oscillations centered on the trajectory of the laser provided the electron energy is sufficiently low ${ }^{15}$. The electron beam will exit the plasma in the laser beam direction provided the electron beam betatron oscillation wavelength is much smaller than the laser centroid oscillation wavelength. In this way laser centroid oscillations can result in large exit angles of both the laser and electron beams.

For both radiation source and high energy physics applications, experiments ${ }^{16}$ and simulations $^{17}$ have shown that the plasma channel density profile must be carefully controlled for efficient guiding and acceleration. Interferometry has been commonly used as a technique for measuring plasma channel profiles in a hydrogen-filled capillary discharge waveguide. Interferometric experiments required the capillary length to be less than a few $\mathrm{mm}$ in the case of longitudinal interferometry (resulting in measurement errors due to end effects), or square, transparent capillaries (and assumptions on symmetry) for transverse interferometry $^{18,19}$. Spot size measurements at the output of the capillary have also been used to deduce channel depth${ }^{20}$, but this technique does not provide the detailed shape of the channel and is sensitive to the mode quality of the probe laser pulse. Hence, accurate diagnostics of plasma channel shape and depth are needed.

In this paper, the centroid location of low-intensity $\left(<10^{14} \mathrm{Wcm}^{-2}\right)$ laser pulses at the output of a plasma channel is used to measure the properties of the channels, including alignment, depth, and profile. Whereas laser pulses of intensity $>10^{18} \mathrm{Wcm}^{-2}$ are used for laser plasma acceleration, the intensity was kept low in these experiments so that non-linear

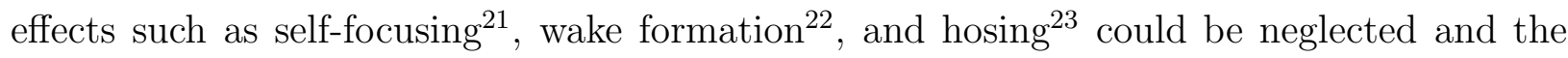
plasma properties measured in a straight-forward way. The technique relies on the fact that a transverse offset of the laser pulse at the entrance of the plasma channel causes the laser beam to undergo transverse oscillations about the channel axis. These oscillations depend on the initial centroid offset and angle with respect to the channel axis, and on the channel properties. As will be shown, measuring the dependence of the exit laser centroid position 
on the input and on channel formation timing provides direct information on the channel matched spot size and profile. This technique reduces the measurement error compared to interferometry and provides information on the transverse density profile. The method also provides increased precision in channel alignment to minimize pointing errors in LPA applications. With knowledge of the channel depth and shape, the effect of laser-capillary alignment on an electron beam can be modeled. For the parameters presented here, $\mu \mathrm{rad}$ jitter in laser pointing (typical of current laser systems) at the input of the waveguide can cause mrad deviations in the laser - and hence potentially electron beam - pointing. Measuring and controlling the channel depth and shape is therefore essential for quantifying and ultimately mitigating laser and electron beam pointing errors.

The paper is organized as follows. Section II describes propagation of a focused laser pulse through a plasma channel. Section III details the experimental layout and presents measurements of the channel depth and shape using the centroid oscillation technique. Conclusions are presented in Section IV.

\section{LASER PROPAGATION IN A PLASMA CHANNEL}

To understand how the propagation of a laser beam through a longitudinally-uniform plasma channel is affected by pointing errors, this section discusses the basic physics for low-intensity laser pulses with power below the critical power for self-focusing, including an initial centroid offset $x_{\mathrm{ci}}$ and injection angle $\theta_{\mathrm{i}}$. It will be shown that the evolution of the centroid position depends only on the density profile and that measuring the dependence of the laser centroid movement at the exit of a plasma channel on the input offset can yield the matched spot size $r_{\mathrm{m}}$ [i.e., the spot size for which the laser beam propagates with constant spot size $\left.r_{\mathrm{s}}(z)=r_{\mathrm{m}}\right]$.

Equations describing the behavior of the laser spot size $r_{\mathrm{s}}(\zeta, z)$ and centroid $x_{\mathrm{c}}(\zeta, z)$ (where $\zeta=z-c t$ is the co-moving variable and $c$ is the speed of light in vacuum) have been derived by analyzing the paraxial wave equation for the envelope of the laser including the effects of a preformed parabolic density channel $n(x, y)=n_{0}+\Delta n\left(x^{2}+y^{2}\right) / r_{\mathrm{m}}^{2}$, where $\Delta n$ is the channel depth at the radius of the matched spot $r_{\mathrm{m}}{ }^{24}$. Consider a laser pulse with a Gaussian intensity profile such that $|a|^{2}=\left(a_{0} r_{0} / r_{\mathrm{s}}\right)^{2} \exp \left(-2 r^{2} / r_{\mathrm{s}}^{2}\right)$, where $a_{0}$ is the normalized laser vector potential given by $a_{0}^{2} \simeq 7.3 \times 10^{-19}(\lambda[\mu \mathrm{m}])^{2} I_{0}\left[\mathrm{Wcm}^{-2}\right], \lambda$ is the 


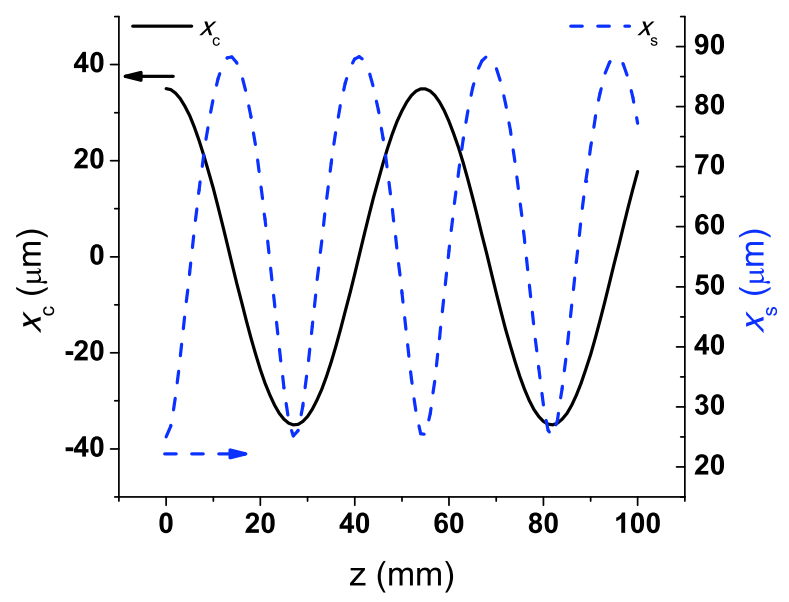

FIG. 1. (color online) Laser centroid offset $x_{\mathrm{c}}$ (solid line) and spot size (dashed line) as a function of propagation distance $z$ in a channel of matched spot size $r_{\mathrm{m}}=47 \mu \mathrm{m}$ for an input centroid offset of $x_{\mathrm{ci}}=35 \mu \mathrm{m}$, input injection angle $\theta_{\mathrm{i}}=0$, and initial spot size $r_{\mathrm{i}}=25 \mu \mathrm{m}$.

laser wavelength, $r_{0}$ is the spot size of the vacuum focus, and $r$ is the distance from the laser centroid $r^{2}=\left(x-x_{\mathrm{c}}\right)^{2}+\left(y-y_{\mathrm{c}}\right)^{2}$ with $x_{\mathrm{c}}$ and $y_{\mathrm{c}}$ the centroid locations in the $x$ and $y$ directions. Then, in the limits $a^{2} \ll 1$ and $k_{\mathrm{p}}^{2} r_{0}^{2} \gg 1$, the evolution of the normalized centroid $\hat{x}_{\mathrm{c}}=x_{\mathrm{c}} / r_{\mathrm{m}}$ is ${ }^{24}$

$$
\left(\frac{\partial^{2}}{\partial \hat{z}^{2}}+\frac{\Delta n}{\Delta n_{\mathrm{c}}}\right) \hat{x}_{\mathrm{c}}=0
$$

and the evolution of the normalized spot size $\hat{r}_{\mathrm{s}}=r_{\mathrm{s}} / r_{\mathrm{m}}$ is

$$
\begin{aligned}
\frac{\partial^{2} \hat{r}_{\mathrm{s}}}{\partial \hat{z}^{2}}-\left(1-\frac{P}{P_{\mathrm{c}}}-\frac{\Delta n}{\Delta n_{\mathrm{c}}} \hat{r}_{\mathrm{s}}^{4}\right) \hat{r}_{\mathrm{s}}^{-3}= & \\
& 4 \hat{r}_{\mathrm{s}} \int_{0}^{\zeta} d \zeta^{\prime} \cos \left[k_{\mathrm{p}}\left(\zeta^{\prime}-\zeta\right)\right] \frac{\partial}{\partial \zeta^{\prime}}\left[\frac{P\left(\zeta^{\prime}\right)}{P_{\mathrm{c}}}\right] .
\end{aligned}
$$

Here $k_{\mathrm{p}}^{2}=4 \pi e^{2} n_{0} / m_{\mathrm{e}} c^{2}$ is the plasma wavenumber, $\hat{z}=z / Z_{\mathrm{m}}, Z_{\mathrm{m}}=k r_{\mathrm{m}}^{2} / 2$ is the matched Rayleigh length, $k=2 \pi / \lambda$ the laser wavenumber, $\Delta n_{\mathrm{c}}=\left(\pi r_{\mathrm{e}} r_{\mathrm{m}}^{2}\right)^{-1}$ denotes the matched channel depth, $r_{\mathrm{e}}$ is the classical electron radius, and $P(\zeta) / P_{\mathrm{c}}=a_{0}^{2} k_{\mathrm{p}}^{2} r_{0}^{2} / 32$ is the laser power normalized to the critical power for relativistic self-focusing. Equations (1) and (2) also assume that the head-to-tail centroid displacements are much smaller than the spot size, i.e., $\left|x_{\mathrm{c}}(\zeta)-x_{\mathrm{c}}\left(\zeta^{\prime}\right)\right| \ll\left|r_{\mathrm{s}}(\zeta)\right|$, which is valid for moderately short laser pulses (laser pulse length $\left.L_{\mathrm{p}} \ll Z_{\mathrm{m}}\right)$ and for $x_{\mathrm{c}} \lesssim r_{\mathrm{s}}$. If the laser pulse length is a significant fraction of the oscillation wavelength $\left(2 \pi Z_{\mathrm{m}}\right)$, then laser hosing will occur ${ }^{23}$, whereas for short pulses the centroid motion, Eq. (1), and spot size motion, Eq. (2), are decoupled. From Eq. (2) the 
condition for matched propagation where the spot size is constant at $r_{\mathrm{s}}=r_{\mathrm{m}}$ is $\Delta n=\Delta n_{\mathrm{c}}$ in the low power limit $\left(P \ll P_{\mathrm{c}}\right)$.

The solution to Eq. (1) is

$$
x_{\mathrm{c}}=x_{\mathrm{i}} \cos \left[\left(k_{\beta \mathrm{c}} z\right)-\varphi\right] \text {, }
$$

where the initial phase $\varphi=\arccos \left(x_{\mathrm{ci}} / x_{\mathrm{i}}\right)$ and oscillation amplitude $x_{\mathrm{i}}=\left[x_{\mathrm{ci}}^{2}+\theta_{\mathrm{i}}^{2} k_{\beta \mathrm{c}}^{-2}\right]^{1 / 2}$ depend on the initial injection centroid offset $x_{\mathrm{ci}}$ and injection angle $\theta_{\mathrm{i}}$, and $k_{\beta \mathrm{c}}=Z_{\mathrm{m}}^{-1}$ is the wavenumber of the laser centroid oscillation about the plasma channel axis assuming $\Delta n=\Delta n_{\mathrm{c}}$. The period of the centroid oscillation does not depend on the laser power but only on the matched spot size of the channel $r_{\mathrm{m}}$.

For $r_{\mathrm{s}}=r_{\mathrm{i}}$ and $d r_{\mathrm{s}} / d z=0$ at the channel entrance $(z=0)$, the spot size evolution given by the solution of Eq. (2) assuming $P \ll P_{\mathrm{c}}$ is

$$
r_{\mathrm{s}}^{2}=\frac{r_{\mathrm{i}}^{2}}{2}\left[1+\frac{r_{\mathrm{m}}^{4}}{r_{\mathrm{i}}^{4}}+\left(1-\frac{r_{\mathrm{m}}^{4}}{r_{\mathrm{i}}^{4}}\right) \cos \left(2 k_{\beta \mathrm{c}} z\right)\right] .
$$

The spot size therefore oscillates between $r_{\mathrm{i}}$ (the value at the channel entrance) and $r_{\mathrm{m}}^{2} / r_{\mathrm{i}}$ at twice the frequency of the centroid oscillation. This is shown in Fig. 1, where the laser centroid (solid line) and the spot size (dashed line) are shown as a function of propagation distance $z$ in a channel of matched spot size $r_{\mathrm{m}}=47 \mu \mathrm{m}$ for an input centroid offset of $x_{\mathrm{ci}}=35 \mu \mathrm{m}$ and initial spot size $r_{\mathrm{i}}=25 \mu \mathrm{m}$. Note that self-focusing can affect the matched spot size when $P \sim P_{\mathrm{c}}$. For example, in the long pulse limit (laser pulse length greater than the plasma wavelength) where self-modulation of the laser pulse occurs, the right-hand side of Eq. (2) is zero $\left(\partial_{\zeta} P / P_{\mathrm{c}} \simeq 0\right)$, and the matched spot size $\left(r_{\mathrm{s}} / r_{\mathrm{m}}\right)^{4}=\left(1-P / P_{\mathrm{c}}\right) \Delta n_{\mathrm{c}} / \Delta n$. This is in contrast to the laser centroid oscillation, which is independent of power.

Figure 2 shows the laser offset normalized by input offset $x_{\mathrm{c}} / x_{\mathrm{ci}}$ (solid line), the laser angle (dotted line), and the spot size (dashed line) at the output of a plasma channel of length $15 \mathrm{~mm}$ as a function of $r_{\mathrm{m}}$ as calculated from Eqs. (3) and (4) for $\theta_{\mathrm{i}}=0, x_{\mathrm{ci}}=10 \mu \mathrm{m}$, and $r_{\mathrm{i}}=80 \mu \mathrm{m}$. For a given centroid offset or laser spot size at the output of the waveguide, more than one solution to the matched spot size may exist.

Determination of the matched spot size $r_{\mathrm{m}}$ using the laser centroid, spot size, or angle can be accomplished by several methods. One approach is to vary the length of the plasma channel and measure the oscillation wavelength for constant laser and plasma conditions. This could be done in gas jet experiments with laser-produced channels ${ }^{25}$, but is more difficult to achieve in the case of a capillary discharge waveguide. For capillary discharge 


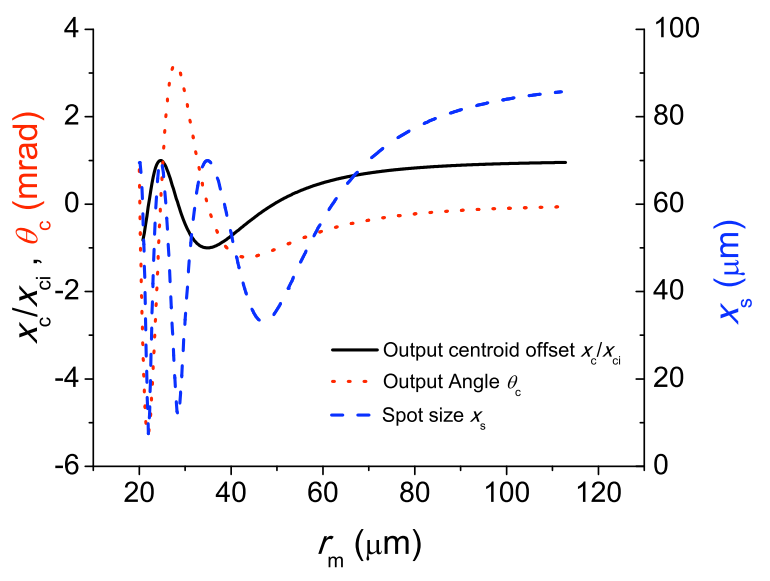

FIG. 2. (color) Laser beam parameters at the output of a plasma channel of length $15 \mathrm{~mm}$ as a function of matched spot size. Normalized laser centroid offset $x_{\mathrm{c}} / x_{\mathrm{ci}}$ (solid line), angle (dotted line), and spot size (dashed line) are shown for an input centroid offset of $x_{\mathrm{ci}}=10 \mu \mathrm{m}$, input injection angle $\theta_{\mathrm{i}}=0$, and $r_{\mathrm{i}}=80 \mu \mathrm{m}$.

waveguides an experimentally more straightforward way is to rely on the assumption that the plasma channel depth continuously decreases on the nanosecond timescale (and the matched spot size increases) after the peak of the discharge ${ }^{26,27}$. The delay between the onset of the plasma channel and arrival of the laser pulse scans the matched spot size from infinity at long delays where there is no discharge current to finite sizes (i.e., scanning right to left in Fig. 2). By measuring the laser spot size this technique has been applied to determine the matched spot size of a hydrogen-filled capillary discharge waveguide ${ }^{20}$. However, these measurements can be difficult to interpret if the laser mode is not Gaussian. For all higher-order modes the spot size and centroid evolution are described by Eqs. (3) and (4) in the low-power limit, and to recover the channel properties requires precise knowledge of the input and output modes. Understanding the mode propagation may require modal decomposition or wavefront analysis. Interaction of the high-order modes with the walls of the capillary may also complicate interpretation. In addition, channel alignment can be accomplished in a straightforward way by scanning the discharge timing (in the same way used in laser spot size measurements described above ${ }^{20}$ ) in combination with scanning the input centroid offset and measuring the output centroid offset. For a properly aligned channel there will be no dependence of the centroid shift on the delay between the laser pulse arrival and initiation of the discharge. 
The centroid oscillation can be used to retrieve not only the channel matched spot size but also its profile. Measurement of channel profile by measuring laser output centroid versus input is illustrated in Fig. 3. The laser centroid shift was calculated for channels with density profiles $n(r)=n_{0}+\Delta n r^{2} / r_{\mathrm{m}}^{2}$ and $n(r)=n_{0}+\Delta n r^{4} / r_{\mathrm{m}}^{4}$ and length $15 \mathrm{~mm}$ for a matched spot of $47 \mu \mathrm{m}$ (the density rise at $r=47 \mu \mathrm{m}$ is held constant). These curves were generated using ZEMAX ${ }^{28}$ by calculating the path of rays offset from the axis. For the case of a parabolic channel, the curve is linear as is expected from Eq. (3), since $z$ (in this case the capillary length) is constant. For a measurement that shows such a linear dependence, the matched spot size obtained from Eq. (3) is

$$
r_{\mathrm{m}}=\sqrt{\frac{2 z}{k\left|\cos ^{-1}\left(d x_{\mathrm{c}} / d x_{\mathrm{ci}}\right)+2 \pi j\right|}}
$$

The integer $j$ can be determined by measuring the response of the laser to discharge timing. It should be noted from Eq. (5) that only the gradient $d x_{\mathrm{c}} / d x_{\mathrm{ci}}$ is used in the calculation of $r_{\mathrm{m}}$ and knowledge of the location of the channel axis is not required. Furthermore, the gradient is independent of the input laser angle $\theta_{\mathrm{i}}$, which adds a constant offset to $x_{\mathrm{c}}$. Since using the slope measurement does not depend on the precise alignment of the capillary, it offers increased accuracy for determining the matched spot size compared with measuring the timing dependence alone.

The centroid displacement technique also provides information on the plasma channel shape. This is illustrated by considering the case of a channel with $r^{4}$ dependence, where rays away from the axis experience a stronger deflection and the curve in Fig. 3 becomes non-linear. By using simple ray-tracing and adjusting the assumed channel shape to match measured data, the channel shape can be retrieved. For the case of a hydrogen-filled capillary discharge waveguide, the density profile for $r<r_{\mathrm{m}}$ has been predicted to be parabolic ${ }^{26,29}$, but rises faster than quadratically near the capillary wall. Interferometry measurements also showed the fast rise in density near the capillary wall, but did not have sufficient sensitivity to measure the profile of the channel for $r<r_{\mathrm{m}}$, since the measurement error in density was on the order of the density rise at $r=r_{\mathrm{m}}{ }^{19}$. Assuming a typical measurement accuracy of $1 \mu \mathrm{m}$ on the laser centroid location, then the measurement error using the centroid technique is only a few $\%$ of the density rise at $r=r_{\mathrm{m}}$, providing the sensitivity needed to measure and improve the channel guiding properties.

It should be noted that increased accuracy can be achieved by simultaneously measuring 


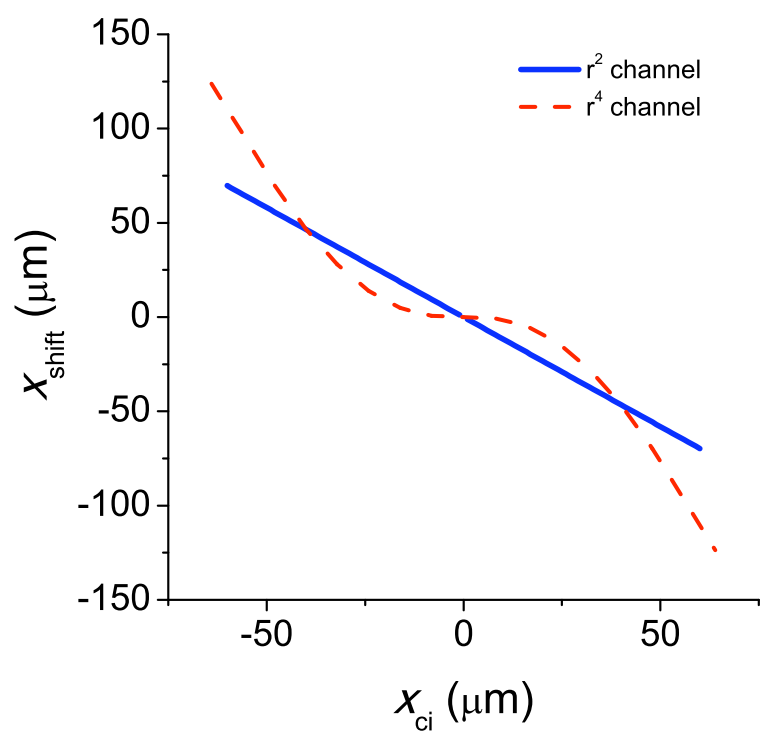

FIG. 3. (color online) Calculated laser centroid shift $x_{\text {shift }}=x_{\mathrm{c}}-x_{\mathrm{ci}}$ at the output of a plasma channel of length $15 \mathrm{~mm}$ as a function of input laser centroid offset for channels of shape $r^{2}$ and $r^{4}$ for $r_{\mathrm{m}}=47 \mu \mathrm{m}$. The channel depth at $r=r_{\mathrm{m}}$ was the same in both cases.

the laser beam exit angle as well as centroid location. In addition the channel depth as measured by the laser centroid shift or angle and the measured spot size at the output of the channel can then be used to calculate the contribution of self focusing using Eq. (2). This is important to quantify since $P \gtrsim P_{\mathrm{c}}$ for most LPA experiments and a laser spot size smaller than $r_{\mathrm{m}}$ may need to be employed for optimum guiding ${ }^{16}$.

In the subsequent two sections, experiments will be described in which the evolution of the plasma channel in a hydrogen-filled capillary discharge waveguide is deduced from measurements of the laser centroid shift at the output of the capillary.

\section{EXPERIMENTAL SETUP AND RESULTS}

The experimental layout is shown in Fig. 4 . Low energy pulses $(<5 \mathrm{~mJ})$ from a Ti:sapphire laser system were focused onto the entrance of a hydrogen-filled capillary discharge waveguide by a $2 \mathrm{~m}$ focal length off-axis parabolic mirror ${ }^{2,3}$. The hydrogen-filled capillary discharge waveguide has been described in detail elsewhere ${ }^{18,19,30}$, along with its use for laser wakefield acceleration of electrons to energies up to $1 \mathrm{GeV}^{2,3}$. 


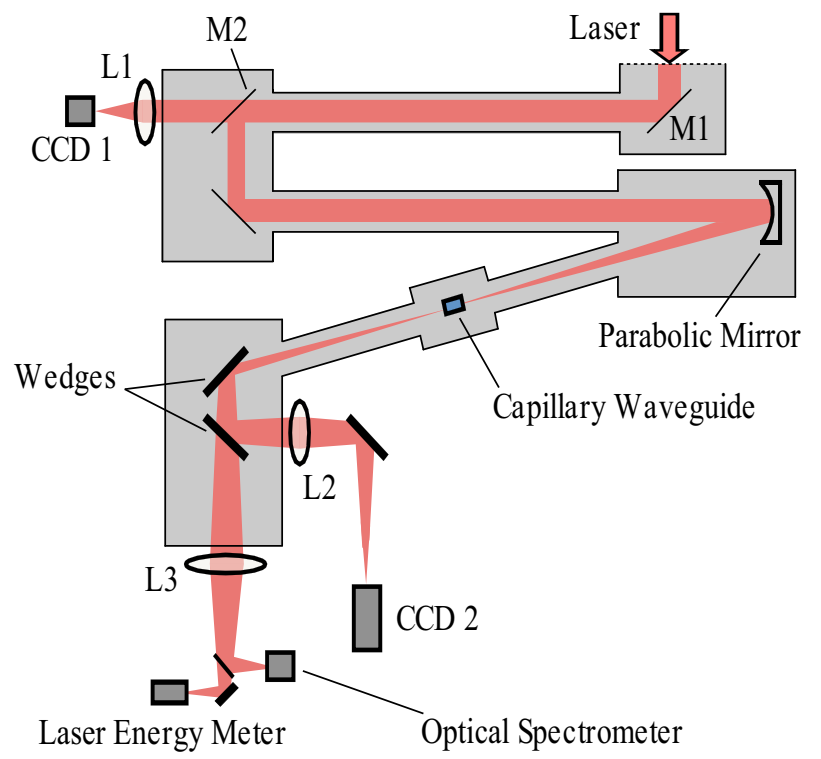

FIG. 4. (color online) The experimental layout used for investigating laser and electron beam propagation in a hydrogen-filled capillary discharge waveguide. Laser pulses were focused onto the entrance of the capillary using an off-axis parabolic mirror. Laser pointing input to the capillary was measured by CCD 1. The spectrum and energy of the laser pulses at the capillary output were measured with a spectrometer and pyroelectric energy meter.

An aperture $2 \mathrm{~cm}$ in diameter was used to increase the effective f-number of the focusing system compared to previous experiments ${ }^{2,3}$, resulting in beams with a spot size of $r_{\mathrm{i}}=$ $70 \mu \mathrm{m}$ at the capillary entrance. The large focal spot allowed for the unguided beam to pass through the $15 \mathrm{~mm}$-long and $300 \mu \mathrm{m}$-diameter capillary with minimal wall interaction since the Raleigh range is $Z_{\mathrm{R}}=19 \mathrm{~mm}$. Thus the centroid location at the output of the capillary when there is no plasma channel $\left(r_{\mathrm{m}}=\infty\right)$ (i.e., when no current flows through the capillary) was the same as when the capillary was removed. The laser energy on target was $0.5 \mathrm{~mJ}$, corresponding to a peak intensity of $5 \times 10^{13} \mathrm{Wcm}^{-2}$ for a pulse length of $120 \mathrm{fs}$. Since this pulse length of $35 \mu \mathrm{m}$ is much shorter than the typical centroid oscillation wavelength of $\sim 10 \mathrm{~mm}$, head-to-tail displacements can be neglected and spot size and centroid motions are decoupled as described in Sec. II. The low laser energy and pulse length longer than for optimized compression ensured that the intensity was below the ionization threshold of hydrogen $\left(\sim 10^{14} \mathrm{Wcm}^{-2}\right)$, avoiding ionization-induced diffraction ${ }^{31}$, and that the power was below the self-focusing limit (i.e., $P \ll P_{\mathrm{c}}$ ), allowing the evolution of the laser spot size to be described by Eq. (4). Since the technique of measuring the centroid oscillation requires 

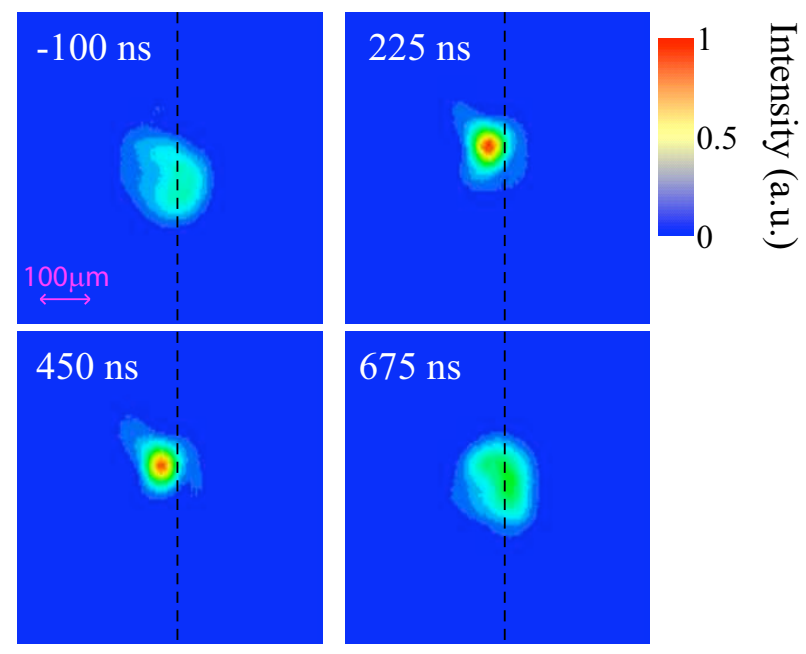

FIG. 5. (color) Typical laser modes at the output of the capillary for the parameters of Fig. 6. The delay between the discharge and laser pulse is shown at the top left of each image. The dashed line is at a constant location in the image. The centroid shift and spot size reduction is clear for delays during the discharge current.

the scanning of the delay $t_{\text {delay }}$ between the laser pulse and onset of the discharge, the laser intensity must be kept low to avoid ionization-induced diffraction. It should be noted that for high power LPA experiments ionization-induced diffraction can be neglected since $t_{\text {delay }}$ can be set such that the plasma channel is fully ionized.

Hydrogen gas filled the capillary to a pressure of 46 Torr via slots located $0.5 \mathrm{~mm}$ from each end. Due to the hydrodynamic evolution of the plasma channel on the tens of nanosecond timescale, varying the delay time between arrival of the laser pulse and initiation of the discharge allowed various channel conditions to be explored. The on-axis density for delays after which a stable plasma channel is formed was calculated to be $1.4 \times 10^{18} \mathrm{~cm}^{-3}$ using the scaling given in Ref. 19.

Laser radiation emerging from the capillary was attenuated by reflection off two optically flat glass wedges. The pulses were refocused by a lens of focal length $500 \mathrm{~mm}$ and diameter $100 \mathrm{~mm}$, allowing for imaging of the output of the capillary onto a 12-bit CCD camera that measures the centroid location and spot size of the laser beam.

The energy of each laser pulse input to the waveguide was determined by a photodiode that was calibrated to the energy on target. The energy of pulses transmitted through the capillary was measured by loosely focusing the portion of the laser beam transmitted 
through the second wedge onto a pyroelectric energy meter that was cross-calibrated to the input photodiode.

The capillary was first roughly aligned by observing the laser beam output mode while varying the capillary transverse position and angle. For optimum alignment translation of the capillary position or angle, by an equal amount in either direction, resulted in an equal change in the portion of laser energy transmitted through the waveguide and of the shift in the laser centroid. The laser centroid shift $x_{\text {shift }}$ was measured by first measuring the laser centroid at the output plane of the capillary without plasma channel. More precise alignment was then achieved by measuring the laser centroid location shift as a function of transverse capillary position $x_{\text {cap }}$. For any $x_{\text {cap }}$, the input offset $x_{\text {ci }}$ can be calculated using $x_{\mathrm{ci}}=x_{\text {shift }}\left(d x_{\text {shift }} / d x_{\text {cap }}\right)^{-1}$, assuming $\theta_{\mathrm{i}}=0$. The capillary was displaced horizontally to create an offset of $x_{\mathrm{ci}}=30 \mu \mathrm{m}$ and example images of the output mode of the laser pulses at the capillary exit for different delays are shown in Fig. 5. At later injection times in the discharge, the laser centroid is shifted and the spot size reduced, consistent with the existence of a plasma channel.

The laser centroid shift due to the plasma channel $x_{\text {shift }}=x_{\mathrm{c}}-x_{\mathrm{ci}}$ at the capillary output for the conditions of Fig. 5 was determined after thresholding the image to $50 \%$, and is plotted versus delay in Fig. 6 along with the discharge current. The centroid shift increases during the first $200 \mathrm{~ns}$ to an approximately constant value for approximately $600 \mathrm{~ns}$. For delays after this, when the discharge current drops below $100 \mathrm{~A}$, the shift begins to return to zero and the spot size increases to the value observed without the capillary in place. This is expected since after the current has decayed there is no plasma channel and the matched spot goes to infinity.

The temporal evolution of the channel can be explained as follows. During the ionization phase, the electron plasma temperature is relatively low and heat transport to the wall is slow, giving rise to a fairly uniform temperature and density. As the majority of the channel reaches full ionization, ohmic heating causes a rapid rise in electron temperature. The ion temperature also rises rapidly as electrons transfer energy via collisions. A temperature gradient is set up as heat is conducted to the wall. The pressure gradient (which follows from the ideal gas law) is rapidly equilibrated via bulk flow from the capillary center to the wall. $\mathrm{By} \simeq 100 \mathrm{~ns}$ the channel has settled to a stable state with a minimum on axis as expected. The stability of the channel for delay between $100 \mathrm{~ns}$ and $600 \mathrm{~ns}$ can be understood 


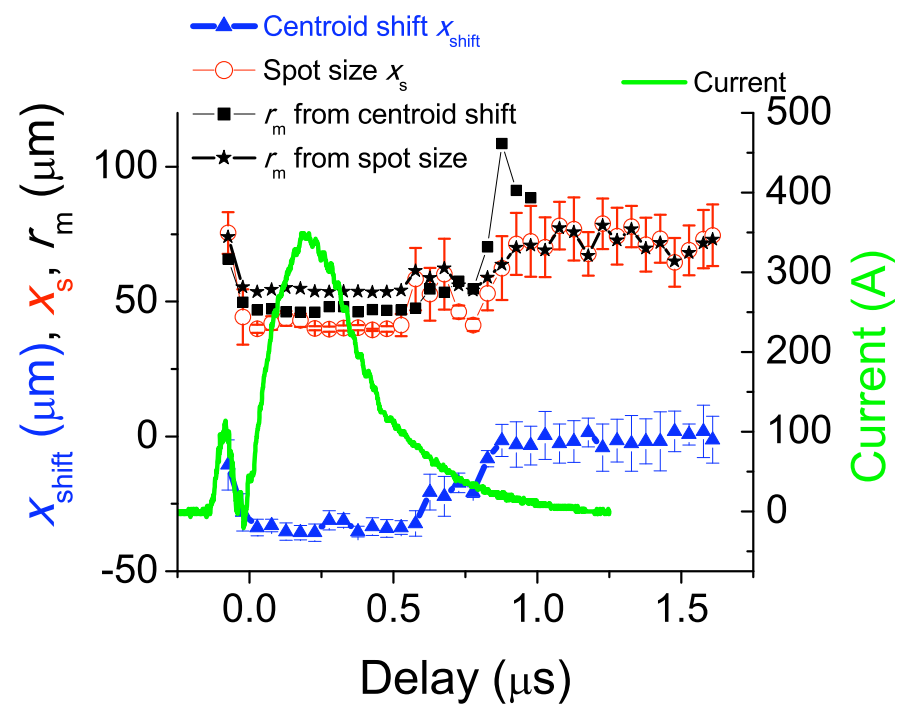

FIG. 6. (color) Measured centroid shift (blue triangles) and spot size $r_{\mathrm{s}}$ in the $x$ direction $\left(x_{\mathrm{s}}\right.$, red circles) as a function of delay between arrival of the laser pulse and onset of the discharge for an offset of $x_{\mathrm{ci}}=-30 \mu \mathrm{m}$ and a capillary of diameter $300 \mu \mathrm{m}$ and length $15 \mathrm{~mm}$. The green line shows the discharge current. The matched spot size as calculated from the laser centroid shift using Eq. (5) is shown by the black squares. The matched spot size as calculated from the laser spot size at the output of the capillary using Eq. (4) is shown by the black stars.

as follows. As the current drops one would expect the electron temperature to decrease. This effect, however, is reduced by the fact that a lower electron temperature gives rise to lower electrical and heat conductivities. This increases heat transferred to the plasma by ohmic dissipation and decreases the energy being conducted to the wall; hence the temperature does not drop as rapidly as one might expect. This was observed in simulations ${ }^{26}$ where a change in current of factor 2 resulted in a change in $r_{\mathrm{m}}$ of less than $4 \%$, explaining why the rms fluctuation in $r_{\mathrm{m}}$ calculated over $400 \mathrm{~ns}$ varied by only $2 \%$.

It should be noted that for the hydrogen-filled capillary discharge waveguide the ablation rate due to the hot plasma is low. The average ablation for a $210 \mu \mathrm{m}$-width square capillary operating with on-axis density of $1.4 \times 10^{18} \mathrm{~cm}^{-3}$ and peak current $300 \mathrm{~A}$ was less than $10 \mu \mathrm{m}$ after 1.3 million shots ${ }^{32}$. This change in capillary radius corresponds to a change in $r_{\mathrm{m}}$ of just $5 \%$.

The matched spot was obtained by scanning backwards in delay (see Fig. 6), which is effectively equivalent to scanning from infinite to finite $r_{\mathrm{m}}$ in Fig. 2. The matched spot can 
then be uniquely determined with the assumption $\theta_{\mathrm{i}}=0$ using Eq. (5) with $d x_{\mathrm{c}} / d x_{\mathrm{ci}}=x_{\mathrm{c}} / x_{\mathrm{ci}}$ and $j=0$. The calculated values for $r_{\mathrm{m}}$ are shown by the black squares in Fig. 6. For delays where the current remains above $100 \mathrm{~A}$, the average matched spot calculated from the centroid shift is $46.9 \pm 0.8 \mu \mathrm{m}$, which is in excellent agreement with $46.5 \mu \mathrm{m}$ given by the scaling derived from simulations ${ }^{33}$. It should be noted that $Z_{\mathrm{m}}=8.6 \mathrm{~mm}$ and $L_{\mathrm{p}}=35 \mu \mathrm{m}$, so the assumption $L_{\mathrm{p}} \ll Z_{\mathrm{m}}$ used in Eqs. (1) and (2) is valid.

The spot size $r_{\mathrm{s}}$ at the output of the capillary was obtained by fitting the intensity profile to the form $\exp \left(-2 r^{2} / r_{\mathrm{s}}^{2}\right)$. The spot size shows the same dependence on delay as the laser centroid shift since the plasma channel length is less than half the laser centroid oscillation wavelength $\lambda_{\beta c}=2 \pi Z_{\mathrm{m}}=54 \mathrm{~mm}$. The matched spot size $r_{\mathrm{m}}$ was calculated from $r_{\mathrm{s}}$ using Eq. (4), (see Fig. 6). The difference between $r_{\mathrm{m}}$ calculated from the spot size (stars in Fig. 6) and centroid (squares in Fig. 6) data is at least in part due the mode not being purely Gaussian. Although Eq. (4) is valid for all higher order modes, the intensity profile at the exit of the plasma channel will, in general, be a complicated sum of these modes. Recovery of $r_{\mathrm{m}}$ from the fitted $\mathrm{rms}$ spot size is then not straightforward and would require precise knowledge of the amplitude and phase of the input laser pulse. This is illustrated in Fig. 6, where finite $r_{\mathrm{m}}$ is obtained from the spot size data for long delays where there is no plasma channel, indicating that there are multiple higher order modes that cannot be well described by the fitted profile. The measured spot size $r_{\mathrm{s}}$ at long delays was the same as when the capillary was removed (i.e., vacuum diffraction), confirming that there was no plasma channel. The centroid technique can also be sensitive to the mode content of the laser pulse. However, Eq. (5) can still be used as long as the mode is symmetric and that there is not significant interaction at the capillary wall. This can be understood by ray analysis, where rays not initially parallel to - but symmetric about - the axis will not affect the centroid location.

Measurement of the channel shape used both the timing scan data and measurement of the output laser centroid location versus input location at fixed delay. Figure 7 shows the output-input data, taken at a constant delay of $t_{\text {delay }}=225 \mathrm{~ns}$ for the same parameters as Fig. 6. The linear dependence implies a parabolic plasma channel over the extent of the matched spot. The matched spot size was calculated to be $r_{\mathrm{m}}=47 \mu \mathrm{m}$ by performing a linear fit to the data and using Eq. (5), where $j=0$ was determined from the fact that the centroid shift undergoes less than half an oscillation in Fig. 6. This is consistent with 


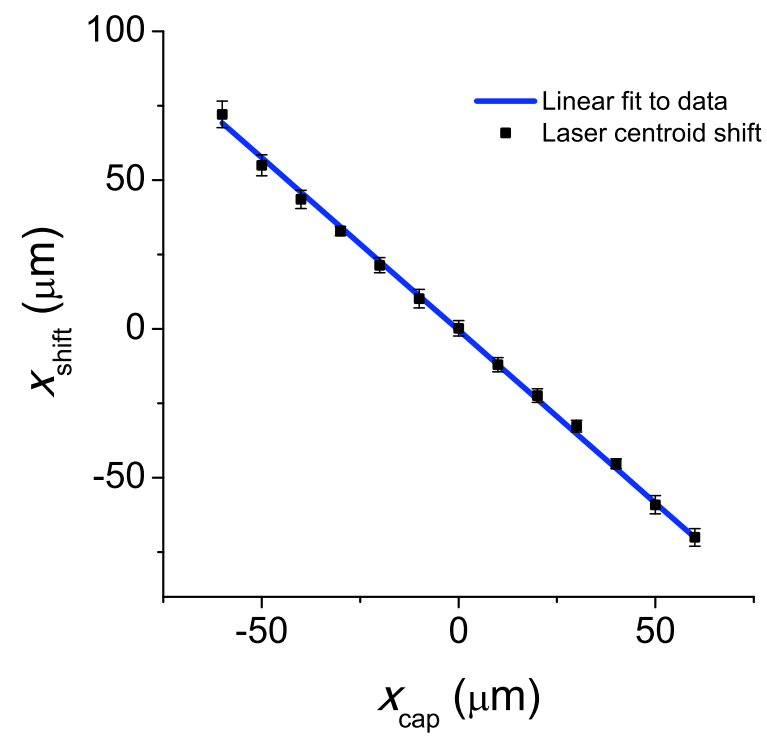

FIG. 7. (color online) Measured centroid shift (squares), as a function of input laser centroid offset for a capillary of diameter $300 \mu \mathrm{m}$ and length $15 \mathrm{~mm}$ and delay $225 \mathrm{~ns}$. The linear fit (solid line) is equivalent to fitting a parabolic shape to the channel.

$r_{\mathrm{m}}=46.5 \mu \mathrm{m}$ given by the scaling derived from simulations ${ }^{33}$. Close to the wall the density has been shown to rise faster than $r^{2}$ since the temperature gradient increases ${ }^{19,26,33}$, which is difficult to probe using this technique since large offset would cause clipping of the beam at the capillary entrance and wall guiding can change the oscillation period. However this effect is not important since for matched guiding there is negligible laser energy close to the wall.

Calculation of $r_{\mathrm{m}}$ using the gradient $d x_{\text {shift }} / d x_{\text {cap }}$ was more accurate than calculation based on a single value of $x_{\text {cap }}$ (as was done for Fig. 6), since the error in initial alignment of $\theta_{\mathrm{i}}$ leads to errors in the determination of $x_{\mathrm{ci}}$. These errors can be reduced by more accurate alignment of the capillary and laser beam, and can be achieved by exploiting the time varying nature of the discharge. For a properly aligned channel there will be no dependence of the centroid shift on the delay time between laser pulse arrival and initiation of the discharge. The alignment error is dominated by the accuracy of centroid determination, which was approximately $5 \mu \mathrm{m}$, limited by the level of magnification of the imaging system for the capillary output. This is significantly better than other techniques that rely on optimizing throughput or modal shape and typically allow for alignment precision to within 
$\sim 20 \mu \mathrm{m}$. With a higher magnification of the imaging system, it is expected that the alignment accuracy of the centroid motion based technique could be lowered to $\sim 1 \mu \mathrm{m}$. This improvement in accuracy of capillary alignment is expected to prove crucial for LPAs since a $\mu \mathrm{m}$ change in alignment can potentially lead to a mrad change in electron beam pointing.

\section{CONCLUSION}

The motion of low-power $\left(P \ll P_{\mathrm{c}}\right)$ laser pulses in a plasma plasma channel for the case of finite offset between the laser and plasma channel axes has been investigated theoretically and experimentally. The laser centroid undergoes oscillations about the plasma channel axis at half the spot size oscillation frequency of a mismatched laser pulse. It was shown that the laser centroid offset at the capillary output due to off-axis injection of the laser pulse could be used to determine the plasma channel depth, and could also be used to accurately deduce the channel shape. Even though the method cannot provide information on the axial electron density, the low-power matched spot can be determined to a high degree of accuracy. The improved accuracy in measuring $r_{\mathrm{m}}$ is important for determining the contribution of self-focusing in laser wakefield experiments (significant when $P \gtrsim P_{\mathrm{c}}$ ), where a matched spot size larger than $r_{\mathrm{m}}$ may be required for optimum guiding. Measurements of the matched spot size and density profile in a hydrogen-filled capillary discharge waveguide using this technique were in excellent agreement with previously published scaling laws ${ }^{33}$ and showed that the channel shape was parabolic within the radius of the matched spot. It was also

found the laser centroid measurements provide a way to align the plasma channel with improved accuracy.

Laser centroid motion can result in electron beam centroid motion. If the betatron wavelength of an injected electron beam is less than the laser centroid oscillation wavelength, which is the case for typical LPA parameters, then the electron beam will track the laser pulse and likewise undergo oscillations in the channel, exiting the plasma in the laser direction. Laser pointing jitter is therefore a mechanism for increasing electron beam pointing jitter in a channel-guided laser plasma accelerator and it might be expected that use of a waveguide would increase electron beam jitter. However, the lowest reported value for the electron beam of $0.7 \mathrm{mrad} \mathrm{rms}$ was achieved through the use of a capillary waveguide ${ }^{8}$. Pointing jitter of 
$1.4 \mathrm{mrad} \mathrm{rms}$ has been achieved in a gas cell ${ }^{10}$, which is several times lower than that typically observed in unguided experiments ${ }^{9}$. Other effects such as the stability and homogeneity of the plasma and laser profiles will need to be investigated to understand the differences in electron beam pointing jitter between the guided and unguided LPA experiments.

In the limit that the laser betatron oscillation wavenumber is greater than that of the electron beam, i.e., $k_{\beta c}>k_{\beta}$, the induced amplitude of the electron beam centroid oscillation is given by $x_{\mathrm{c}}=\left(k_{\beta} / k_{\beta \mathrm{c}}\right) x_{\mathrm{i}}$ where $x_{\mathrm{i}}$ is the laser centroid amplitude. This electron beam centroid oscillation may lead to emittance growth through phase mixing processes, provided the mixing length is less than the stage length. To keep the growth in emittance small, the ratio of $x_{\mathrm{c}}$ to the matched beam radius must be small, which is a restrictive constraint since the matched beam radius is typically small as a result of the strong focusing forces of the wake. Minimizing $x_{\mathrm{c}}$ requires minimizing $k_{\beta}$ which can be achieved by decreasing the wake focusing forces in the linear regime through, for example shaping of the transverse laser mode $^{34}$. For high energy electron beams coupled into subsequent LPA stages, the increase in betatron wavelength $\left(k_{\beta} \propto \gamma^{-1 / 2}\right)$ reduces the impact of the laser beam centroid movement on the electron beam pointing and any emittance growth.

To minimize laser and electron beam pointing errors in laser plasma accelerators, several other strategies can be followed. Laser beam pointing control will need to be improved to well below the $\mu$ rad level to ensure that $x_{\mathrm{i}}$ is minimized. For the parameters presented here, $\mu$ rad jitter in laser pointing (typical of current laser systems) at the plasma channel input can cause mrad deviations in the laser angle during propagation through the plasma

channel. Since the electron beam will follow the laser beam trajectory (provided the electron beam betatron oscillation wavelength is much smaller than the laser centroid oscillation wavelength), the plasma channel length should be tailored to allow for an integer number of betatron oscillations. This ensures that even in the presence of spatial offsets of the laser beam centroid, angular deflections at the exit of the plasma channel will be near or at zero value.

\section{ACKNOWLEDGMENTS}

The authors would like to thank N. Matlis, G. Plateau, J. van Tilborg, and E. CormierMichel for useful discussions. We appreciate contributions from D. Panasenko, D. Syversrud, 
and N. Ybarrolaza. This work was supported by the Director, Office of Science, Office of High Energy Physics, of the U.S. Department of Energy under Contract No. DE-AC0205CH11231, and the Defense Advanced Research Projects Agency (DARPA).

\section{REFERENCES}

${ }^{1}$ E. Esarey, C. B. Schroeder, and W. P. Leemans, Rev. Mod. Phys. 81, 1229 (2009).

${ }^{2}$ W. P. Leemans, B. Nagler, A. J. Gonsalves, C. Tóth, K. Nakamura, C. G. R. Geddes, E. Esarey, C. B. Schroeder, and S. M. Hooker, Nat. Phys. 2, 696 (2006).

${ }^{3}$ K. Nakamura, B. Nagler, C. Tóth, C. G. R. Geddes, C. B. Schroeder, E. Esarey, W. P. Leemans, A. J. Gonsalves, and S. M. Hooker, Phys. Plasmas 14, 056708 (2007).

${ }^{4}$ W. P. Leemans, C. G. R. Geddes, J. Faure, C. Tóth, J. van Tilborg, C. B. Schroeder, E. Esarey, G. Fubiani, D. Auerbach, B. Marcelis, M. A. Carnahan, R. A. Kaindl, J. Byrd, and M. C. Martin, Phys. Rev. Lett. 91, 074802 (2003).

${ }^{5}$ F. Grüner, S. Becker, U. Schramm, M. Fuchs, R. Weingartner, D. Habs, J. Meyer-ter Vehn, M. Geissler, M. Ferrario, L. Serafini, B. van der Geer, H. Backe, W. Lauth, and S. Reiche, Appl. Phys. B-Lasers O. 86, 431 (2007).

${ }^{6}$ P. Tomassini, A. Bacci, J. Cary, M. Ferrario, A. Giulietti, D. Giulietti, L. A. Gizzi, L. Labate, L. Serafini, V. Petrillo, and C. Vaccarezza, IEEE Trans. Plasma Sci. 36, 1782 (2008). ${ }^{7}$ W. P. Leemans and E. Esarey, Phys. Today 62, 44 (2009).

${ }^{8}$ A. J. Gonsalves, E. Esarey, C. G. R. Geddes, W. P. Leemans, C. Lin, K. Nakamura, D. Panasenko, C. B. Schroeder, and C. Tóth, in PAC09 (JACOW, Vancouver, 2009).

${ }^{9}$ S. P. D. Mangles, A. G. R. Thomas, O. Lundh, F. Lindau, M. C. Kaluza, A. Persson, C. G. Wahlstrom, K. Krushelnick, and Z. Najmudin, Phys. Plasmas 14, 056702 (2007).

${ }^{10}$ J. Osterhoff, A. Popp, Z. Major, B. Marx, T. P. Rowlands-Rees, M. Fuchs, M. Geissler, R. Hörlein, B. Hidding, S. Becker, E. A. Peralta, U. Schramm, F. Grüner, D. Habs, F. Krausz, S. M. Hooker, and S. Karsch, Phys. Rev. Lett. 101, 085002 (2008).

${ }^{11}$ C. B. Schroeder, C. G. R. Geddes, C. Tóth, E. Esarey, and W. P. Leemans, in 13th Advanced Accelerator Concepts Workshop, edited by C. B. Schroeder, W. P. Leemans, and E. Esarey (AIP Conference Proceedings, Santa Cruz, California, 2008) Vol. 1086, p. 208.

${ }^{12}$ D. Panasenko, A. J. Shu, C. B. Schroeder, A. J. Gonsalves, K. Nakamura, N. H. Matlis, E. Cormier-Michel, G. R. Plateau, C. Lin, C. Tóth, C. G. R. Geddes, E. Esarey, and W. P. 
Leemans, in 13th Advanced Accelerator Concepts Workshop, edited by C. B. Schroeder, W. P. Leemans, and E. Esarey (AIP Conference Proceedings, Santa Cruz, CA, 2008) Vol. 1086, p. 215.

${ }^{13}$ G. Dugan, in 11th Advanced Accelerator Concepts Workshop (AIP Conference Proceedings, Stony Brook, New York, 2004) Vol. 737, p. 29.

${ }^{14}$ A. Popp, J. Osterhoff, Z. Major, R. Horlein, M. Fuchs, R. Weingartner, T. P. RowlandsRees, J. Vieira, M. Marti, R. A. Fonseca, L. O. Silva, S. M. Hooker, F. Grüner, F. Krausz, and S. Karsch, "All-optical steering of laser-wakefield-accelerated electron beams," Submitted to Phys. Rev. Lett.

${ }^{15}$ E. Esarey, B. A. Shadwick, P. Catravas, and W. P. Leemans, Phys. Rev. E 65, 056505 (2002).

${ }^{16}$ C. G. R. Geddes, C. Tóth, J. van Tilborg, E. Esarey, C. B. Schroeder, D. Bruhwiler, C. Nieter, J. Cary, and W. P. Leemans, Nature 431, 538 (2004).

${ }^{17}$ E. Cormier-Michel, C. G. R. Geddes, E. Esarey, C. B. Schroeder, D. L. Bruhwiler, K. Paul, B. Cowan, and W. P. Leemans, in 13th Advanced Accelerator Concepts Workshop, edited by C. B. Schroeder, W. P. Leemans, and E. Esarey (AIP Conference Proceedings, Santa Cruz, California, 2008) Vol. 1086, p. 297.

${ }^{18}$ D. J. Spence and S. M. Hooker, J. Opt. Soc. Am. B 17, 1565 (2000).

${ }^{19}$ A. J. Gonsalves, T. P. Rowlands-Rees, B. H. P. Broks, J. J. A. M. van der Mullen, and S. M. Hooker, Phys. Rev. Lett. 98, 025002 (2007).

${ }^{20}$ P. S. Antsiferov, M. R. Akdim, and H. T. van Dam, Rev. Sci. Instrum. 78, 123107 (2007).

${ }^{21}$ E. Esarey, P. Sprangle, J. Krall, and A. Ting, IEEE J. Quantum Electron. 33, 1879 (1997).

${ }^{22}$ T. Tajima and J. M. Dawson, Phys. Rev. Lett. 43, 267 (1979).

${ }^{23}$ C. Ren and W. B. Mori, Phys. Plasmas 8, 3118 (2001).

${ }^{24}$ P. Sprangle, J. Krall, and E. Esarey, Phys. Rev. Lett. 73, 3544 (1994).

${ }^{25}$ P. Volfbeyn, E. Esarey, and W. P. Leemans, Phys. Plasmas 6, 2269 (1999).

${ }^{26}$ B. H. P. Broks, K. Garloff, and J. J. A. M. van der Mullen, Phys. Rev. E 71, 016401 (2005).

${ }^{27}$ B. H. P. Broks, W. Van Dijk, J. J. A. M. van der Mullen, A. J. Gonsalves, T. P. RowlandsRees, and S. M. Hooker, Phys. Plasmas 14, 023501 (2007).

${ }^{28}$ ZEMAX, http://www.zemax.com, 3001 112th Avenue NE, Suite 202, Bellevue, WA 980048017 USA. 
${ }^{29}$ N. A. Bobrova, A. A. Esaulov, J. I. Sakai, P. V. Sasorov, D. J. Spence, A. Butler, S. M. Hooker, and S. V. Bulanov, Phys. Rev. E 6501, 016407 (2002).

${ }^{30}$ A. Butler, D. J. Spence, and S. M. Hooker, Phys. Rev. Lett. 89, 185003 (2002).

${ }^{31}$ W. P. Leemans, C. E. Clayton, W. B. Mori, K. A. Marsh, P. K. Kaw, A. Dyson, C. Joshi, and J. M. Wallace, Phys Rev A 46, 1091 (1992).

${ }^{32}$ A. J. Gonsalves, Investigation of a Hydrogen-filled Capillary Discharge Waveguide for Laser-driven Plasma Accelerators, Ph.D. thesis, University of Oxford (2006).

${ }^{33}$ B. H. P. Broks, W. van Dijk, and J. J. A. M. van der Mullen, J. Phys. D Appl. Phys. 39, 2377 (2006).

${ }^{34}$ E. Cormier-Michel, E. Esarey, C. G. R. Geddes, C. B. Schroeder, K. Paul, P. Mullowney, J. Cary, and W. P. Leemans, "Control of focusing fields in laser-plasma accelerators using higher-order modes," Submitted to Phys. Rev. E. 


\section{Disclaimer}

This document was prepared as an account of work sponsored by the United States Government. While this document is believed to contain correct information, neither the United States Government nor any agency thereof, nor The Regents of the University of California, nor any of their employees, makes any warranty, express or implied, or assumes any legal responsibility for the accuracy, completeness, or usefulness of any information, apparatus, product, or process disclosed, or represents that its use would not infringe privately owned rights. Reference herein to any specific commercial product, process, or service by its trade name, trademark, manufacturer, or otherwise, does not necessarily constitute or imply its endorsement, recommendation, or favoring by the United States Government or any agency thereof, or The Regents of the University of California. The views and opinions of authors expressed herein do not necessarily state or reflect those of the United States Government or any agency thereof or The Regents of the University of California. 